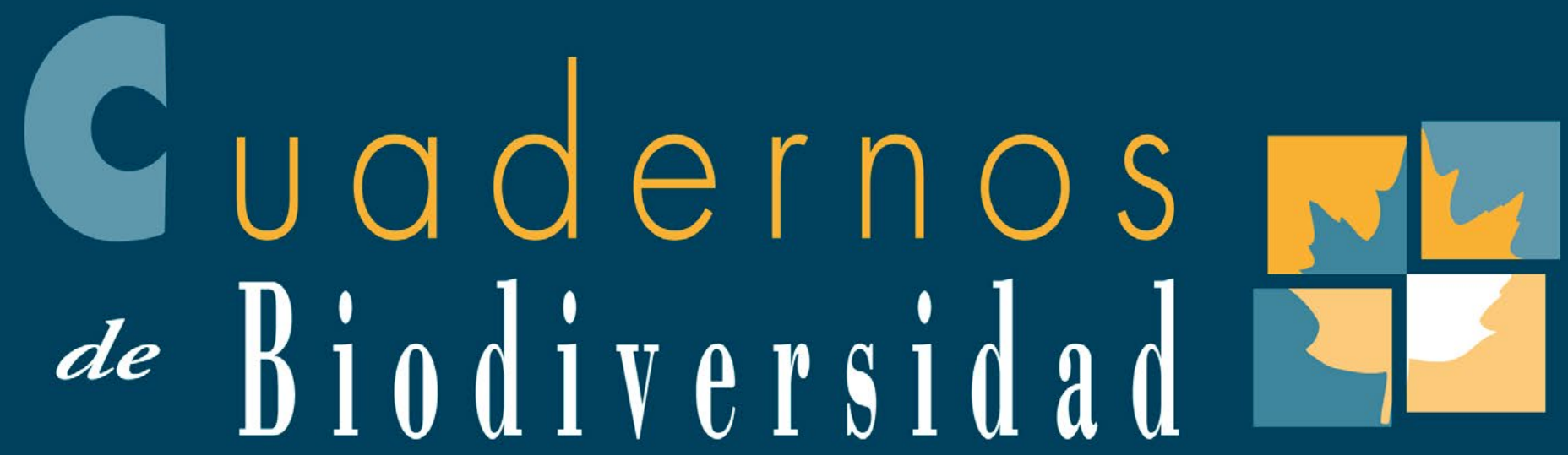

Cuadernos de biodiversidad número 48 • 2015
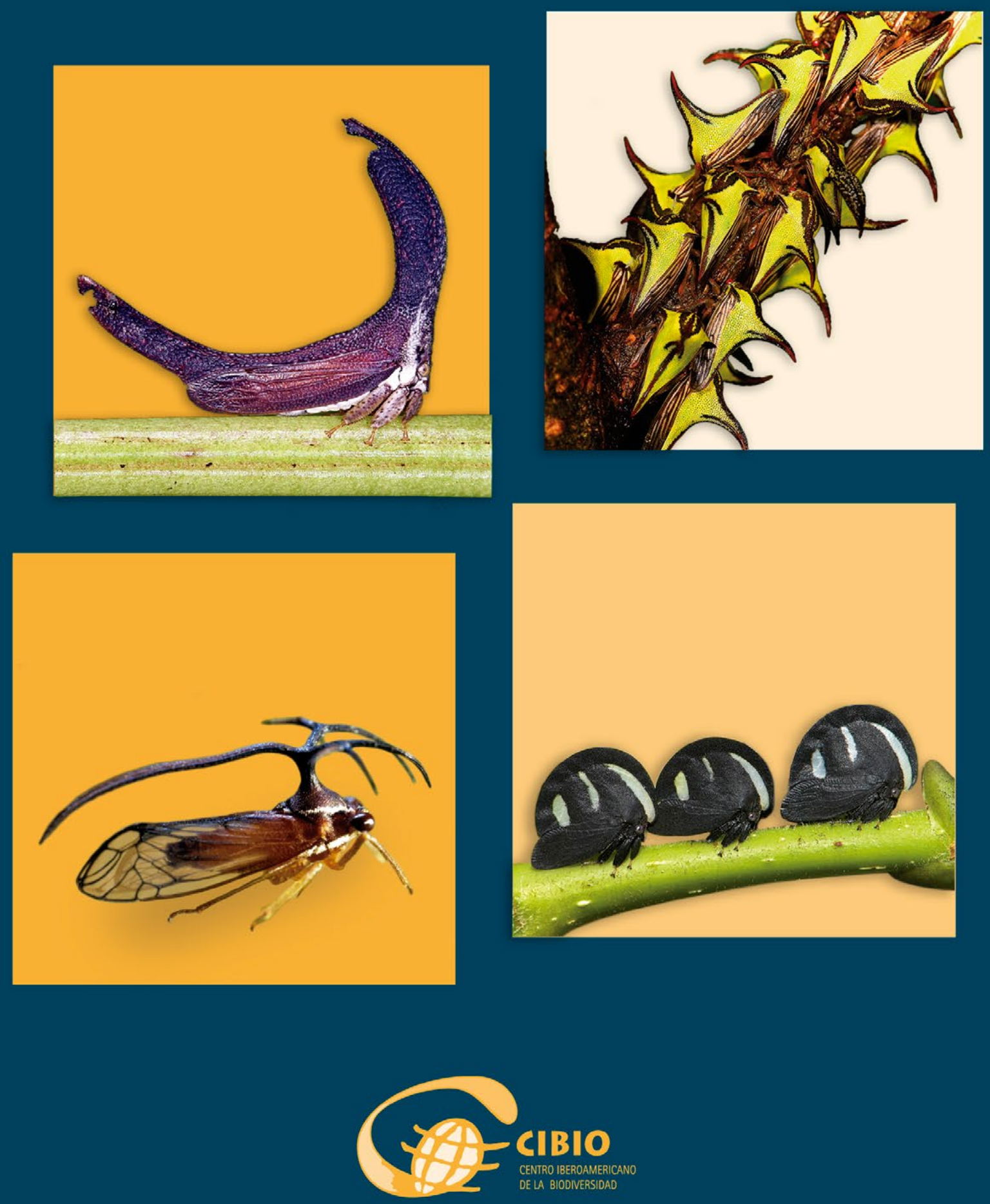

Universitat d'Alacant
Universidad de Alicante 


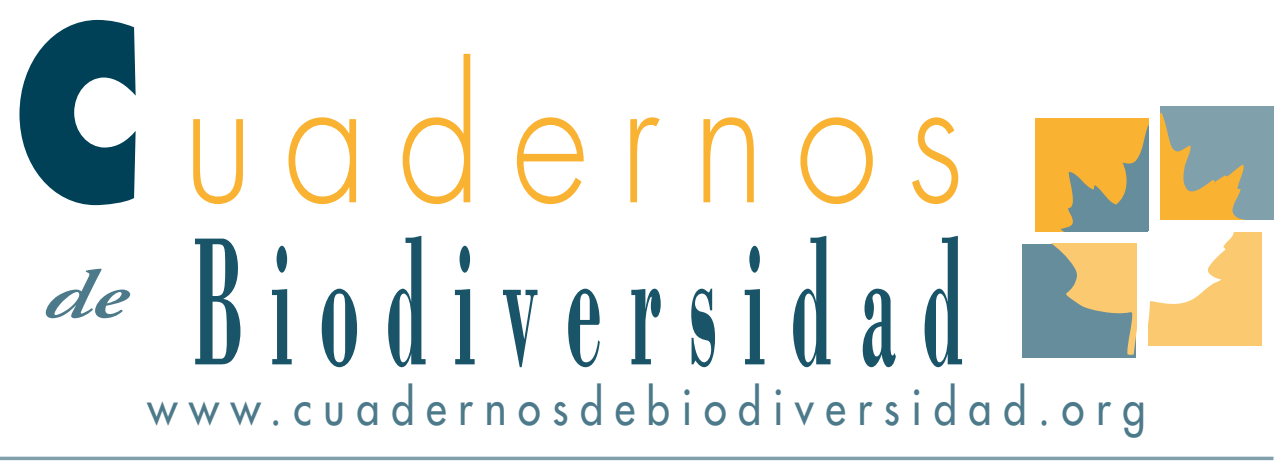

\section{Biodiversidad y cultura cafetalera en México}

\section{A. Contreras Hernández ${ }^{1}$, M. L. Osorio Rosales ${ }^{1}$}

i Instituto de Ecología A. C. Carretera Antigua a Coatepec No 35 i, Col. El Haya. C. P. 9io7o, Xalapa, Veracruz, México E-mail armando.contreras@inecol.mX

\section{ABSTRACT}

Coffee is grown in agro-ecosystems designed by humans in tropical rain forests. Under diversified shade coffee retains much of the structure and functioning of forest ecosystems. After cereals, coffee is the agricultural product moving major currencies in international markets. It is produced in 50 countries by indigenous and peasants. In order to evaluate the coffee culture of central Veracruz region in Mexico, coffee production systems were studied and social organization manners were identified. The project had a transdisciplinary design. The network consists of 100 scholars, 10 organizations of producers and 109 companies in 52 municipalities. In four years (2010-2013), 254 meetings were held with the participation of 6,400 persons. Each activity was thought looking at farmers, their business project and its own culture.

Key words: Agroecosystems, coffee culture, transdisciplinary, resource management, Veracruz

\section{RESUMEN}

El café se produce en agro-ecosistemas diseñados por los seres humanos, a partir de bosques tropicales húmedos. El cafetal bajo la sombra conserva una gran parte de la estructura y funcionamiento de los ecosistemas boscosos. Después de los cereales, el café es el producto agrícola que mayores divisas mueve en el mercado internacional. Se produce en 50 países, principalmente por indígenas y campesinos. Con el objeto de valorar la cultura cafetalera del centro de Veracruz en México, se estudiaron los sistemas de producción de café y se identificaron las formas de organización social. El proyecto ha tenido un diseño transdisciplinario. La red de este estudio está formada por 100 académicos, 10 organizaciones de productores y 109 empresas en 52 municipios. En cuatro años (2010-2013) se realizaron 254 eventos con 6.400 participaciones. Cada actividad se realizó teniendo en cuenta a los productores, su proyecto de empresa y su cultura.

Pallabras clave: agroecosistemas, cultura cafetalera, transdisciplina, manejo de recursos, Veracruz 


\section{INTRODUCCIÓN Y METODOLOGÍA}

El café en México es un cultivo importante que ha sufrido a lo largo del tiempo, vaivenes de productividad y de precios. Se cultiva en 806.000 hectáreas, de las cuales el $83 \%$ son cafetales bajo sombra, con baja productividad, menos de 8 quintales por hectárea (Manson et al., 2008). En los últimos 25 años el café ha sido el principal producto de exportación agrícola. El sector integra 481.000 productores en 4.557 comunidades de 12 estados de México, en orden de importancia -Chiapas, Puebla, Veracruz, Oaxaca, Guerrero, Hidalgo, San Luis Potosí, Nayarit, Jalisco, Colima, Tabasco y Querétaro- $(\mathrm{UACH}$, 2005; Escamilla, 2007; Boege, 2008). La caficultura genera un promedio anual de 700.000 empleos, de los cuales dependen por lo menos 2.3 millones de personas (Nolasco, 1985; Moguel \&Toledo, 1999), ocupando México el octavo lugar del mundo y aporta el $4 \%$ a la producción mundial.

Los cafetales coinciden en altitud con los bosques tropicales, en particular con el bosque mesófilo de montaña (BMM) (Challenger, 1998). Varios estudios ecológicos evidencian que la estructura vegetal de los cafetales y otros sistemas agroforestales puede ser parecida a la del BMM, y por ello pueden albergar una gran diversidad de especies y proporcionar servicios ecosistémicos (Perfecto et al., 1996; Moguel \& Toledo, 1999; Schroth et al., 2004; Manson et al., 2008). La distribución de los cafetales en las partes altas de las cuencas los hace importantes por los servicios ambientales que suministran, y su mal manejo puede impactar negativamente, por ejemplo, la captación y purificación de agua, el control de erosión de suelos y azolve de los ríos, así como la regulación de desastres naturales como deslaves e inundaciones (Stadtmüller \& Agudelo, 1990; Myers, 1997; Bruijnzeel, 2001; Bubb et al., 2004). Aunque el BMM ocupa menos del 1\% de la superficie de México, contiene entre 10 y $12 \%$ de las especies de plantas y animales que existen en el país (Ramamoorthy et al., 1993; Rzedowski, 1996), por lo que es uno de los ecosistemas más importantes y amenazados. Para comprender la regionalización de los sistemas de producción agrarios es necesario reconocer los impactos generados por los procesos de trabajo del producto mismo, el uso de los recursos naturales y la organización social.

Las regiones cafetaleras de Veracruz se definieron hace varias décadas y actualmente no sabemos de qué modo la crisis del café modificó las zonas actuales de producción. Por lo anteriormente mencionado, es pertinente analizar la evolución de las regiones cafetaleras, y redefinir las regiones ya reconocidas según sus características nuevas (De Grammont \& Tejeda, 1996). Hacen falta estudios interdisciplinarios sustentados en los marcos conceptuales de la agroecología y sociología rural que ayuden a los productores a identificar y superar los retos asociados con el desarrollo de estrategias de manejo sustentables en el sector agrícola (Perfecto et al., 2005; Gordon et al., 2007).

Los estudios de biodiversidad exigen inventarios finos de las especies, modelos de las funciones y procesos ecológicos de los ecosistemas. Para el estudio de los cafetales el proyecto Biocafé, antecesor del proyecto Café In Red, se llevó a cabo en el Instituto de Ecología A. C., (INECOL), entre los años 2003 y 2008. Este proyecto estudió los cambios en la diversidad biológica de 13 grupos taxonómicos que habitan las fincas de la región central de Veracruz (Coatepec y Huatusco). Se analizaron 18 fincas de café y tres fragmentos de BMM con el fin de aproximarse a un conocimiento integral del cafetal (Manson et al., 2008). Además, se reconoció la existencia de un gradiente de intensificación del manejo de los cafetales sobre la capacidad de estos agroecosistemas para conservar la biodiversidad (HernándezMartínez et al., 2009). Se incluyeron también varios estudios sobre los cambios en el funcionamiento de estos agroecosistemas a través del monitoreo de servicios ambientales clave proporcionados por las fincas (e. g. secuestro de carbono, polinización, captación de agua y mantenimiento de la fertilidad del suelo). Este estudio logró a través de un enfoque multi-taxonómico, interdisciplinario y de múltiples escalas temporales y espaciales, la formación de un inventario de 2.200 especies que demostró que aún se conserva el $87 \%$ de la biodiversidad del BMM en los cafetales (Manson et al., 2008). 
Los objetivos de esta investigación se alcanzaron trabajando directamente con los productores de café usando un modelo de investigación-acción (Bacon et al., 2005; Contreras \& Hernández, 2008), con la intención de generar conocimiento básico y aplicado a corto plazo, para mejorar el balance entre la conservación del patrimonio biológico de México y el bienestar socio-económico de los productores de café.

A partir del análisis del paisaje cafetalero se generaron nuevas interpretaciones de las unidades de producción, de las estrategias de manejo y de las formas de enfrentar los retos económicos ante la crisis del precio del café. Se destaca que en Veracruz los potreros, cultivos y zonas urbanas ocupan un $72 \%$ de la superficie estatal, y sólo se mantenía un 8.8\% de vegetación natural, sin que existieran indicios de frenar el cambio de uso del suelo. En algunas zonas la distribución del BMM fue remplazada por cafetales, y en el centro del estado es la superficie arbolada continua más importante. La región tiene la infraestructura necesaria para impulsar el desarrollo del sector en el centro de Veracruz.

Otras aportaciones importantes son los constantes intercambios con casi 300 participantes del proyecto, y el fortalecimiento de la autonomía de las 12 organizaciones a través de 30 encuentros culturales y 9 talleres (Contreras, 2008). Considerándose que uno de los mayores logros del proyecto Biocafé fue facilitar la creación del Centro Agroecológico del Café, A.C. (CAFECOL), una institución privada conformada por productores en alianza con instituciones académicas. Con la experiencia del proyecto Biocafé, la formación del CAFECOL y la firma de un primer convenio de colaboración entre instituciones académicas, se formó un grupo líder para la construcción del proyecto subsiguiente Café In Red. Se realizó una convocatoria entre académicos con los criterios siguientes:

a) visión de red de colaboración con acuerdos de trabajo,

b) asumir compromisos con responsabilidad y honestidad entre los actores sociales participantes, c) mostrar una disciplina en el trabajo,

d) apreciar a los cafetales de forma integral,

e) tener un enfoque agroecológico,

f) incluyente, diverso y actuar de forma intersectorial,

g) fomentar la innovación y

h) que sus prácticas ecológicas, sociales y económicas fomenten la sustentabilidad de la cafeticultura. El grupo establecido, se acercó a los actores sociales y construyó los vínculos necesarios para echar a andar el proyecto. En 2010 se obtuvo el apoyo del Fondo Institucional de Fomento Regional para el Desarrollo Científico, Tecnológico y de Innovación (FORDECyT) del Consejo Nacional de Ciencia y Tecnología (CONACYT), con una apoyo del 80\%, la contraparte $(20 \%)$ fue aportada por el consorcio integrado por el INECOL, CAFECOL, Centro Internacional de Investigación, Innovación, Desarrollo y Transferencia de Tecnología Alfredo Cabańas A.C. (CIIIDTTAC) y el Instituto Nacional de Investigaciones Forestales, Agrícolas y Pecuarias (INIFAP).

El fundamento metodológico del proyecto es el trabajo transdisciplinario (Morin \& Brigitte, 1993; Nicolescu, 2008; Núñez et al., 2011), donde los actores sociales intervienen conforme a un objetivo común, lo cual considera la generación de información y su transferencia. El desarrollo de capacidades locales en pro de la conservación de los recursos naturales regionales, como resultado del interactuar de los miembros de la red y el fortalecimiento de la institucionalidad local. En suma, se propicia densificar la energía de la región a favor del bienestar colectivo incluyente y equitativo (Zermeño, 2010).

Se integraron en la red miembros de diferentes regiones cafetaleras de Veracruz y de otros estados vecinos, con fines de alimentar, cooperar y mantener fuentes de información. Estas fuentes se traducen en acciones específicas como son: mantener comunicación a través de boletines de información e internet, fomentar vínculos de colaboración con representantes gubernamentales federales (Sistema- 
Producto Café y Fondo Nacional Cafetalero) y de los estados cafetaleros para acompañar el trabajo de la red. De esta manera, se crearon efectos sinérgicos. La participación de otras regiones es crucial en el futuro de la red, ya que, a lo lardo del proyecto y una vez validados los resultados; se fomentó la transferencia de conocimientos y tecnología.

Las investigaciones agroecológicas adquieren mayor significado si se contrastan con estudios de la dinámica social, históricos, económicos, y políticos de la región. Los integrantes de la red tienen la experiencia de haber desarrollado estudios disciplinarios y para lograr mejores resultados se incorporaron enfoques integrales como el ordenamiento territorial, el manejo integrado de cuencas, el desarrollo de regiones medias y el fortalecimiento de capacidades locales de gestión, en suma; la cultura cafetalera. Todo esto, con el fin de identificar las mejores formas extractivas del capital y las estrategias locales que posibilitan relaciones más equilibradas entre los monopolios externos, las burguesías regionales y los procesos locales. Se quiere demostrar que la investigación es una buena inversión de mediano y largo plazo (Drucker, 2010) para regiones como el centro de Veracruz que, prácticamente, está en el abandono como el resto del campo mexicano (De Grammont, 2005). En el corto plazo la investigación-acción y la participación social pueden ser la palanca para incidir en la reorganización regional.

El objetivo de Café In Red es consolidar una red estratégica de grupos de productores, centros de investigación e instancias gubernamentales para la región centro de Veracruz (Figura 1). Los objetivos específicos son: 1) fomentar un nuevo clúster de innovación con su propio sistema de inteligencia para el desarrollo de capacidades agroecológicas dirigidas a la producción diversificada y sustentable de café de calidad, 2) identificar nichos especializados de mercado y autosuficiencia alimentaria, y 3) apoyar la conservación y restauración de los ecosistemas naturales mediante el establecimiento de estrategias de pagos por servicios ambientales y propuestas de políticas públicas que mejoren las condiciones de vida de los cafeticultores. El proyecto está conformado por cinco estrategias operativas.

\section{SISTEMA DE INTELIGENCIA (SIGCAFÉ)}

Reorganiza y articula el sector cafetalero para impulsar el desarrollo a través de su Sistema de Inteligencia (SI), sus funciones son: a) la organización del grupo operativo; b) diseñar un modelo de regionalización que favorezca la producción de café de calidad con bases agroecológicas y la investigación participativa; c) difundir las propuestas de las líneas estratégicas de la red para la conservación ambiental y la producción sustentable, en el marco de gestión de las cuencas hidrológicas, mediante el fomento de los servicios ambientales y la conservación de la biodiversidad; d) indagar el impacto ambiental de los agroecosistemas cafetaleros e integrarlos a un sistema de información geográfica; e) elaborar propuestas de política pública en los tres órdenes de gobierno (municipal, estatal y nacional); f) dinamizar los procesos locales que mejoren las condiciones de vida de los participantes, con énfasis en las clases más vulnerables de la cadena de valor cafetalera y g) desarrollar un programa de cultura cafetalera.

\section{SERVICIOS AMBIENTALES Y PROTECCIÓN DEL AMBIENTE (SACAF)}

Fomenta la cuantificación, valoración y conservación de los servicios ecosistémicos proporcionados por fincas de café de sombra en el centro de Veracruz con el fin de: a) promover y facilitar la gestión de Pagos por Servicios Ambientales (PSA); b) facilitar la participación de los productores de café en los nuevos espacios de mercados certificado que ofrecen precios más elevados y estables, así como mayores tasas de crecimiento que los mercados convencionales; c) contribuir con información científica y técnica para las propuestas de ordenamiento territorial, cambio climático y conservación del ambiente; y d) contribuir con capacitación, proyectos demostrativos e investigación aplicada en los temas: fertilidad del suelo, contaminación de aguas originada por la producción del café; evaluación de los servicios ambientales (polinización del cafeto, secuestro de carbono en el BMM de las fincas, zonas hidrológicas claves y remanentes de vegetación natural); certifica- 


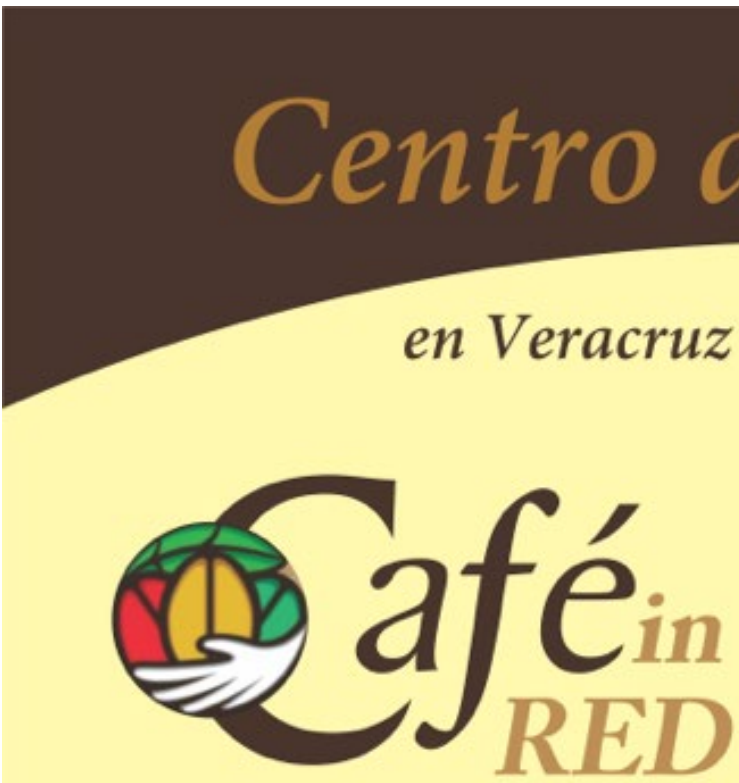

Apoyar la producción de café es parte escencial de su misión...

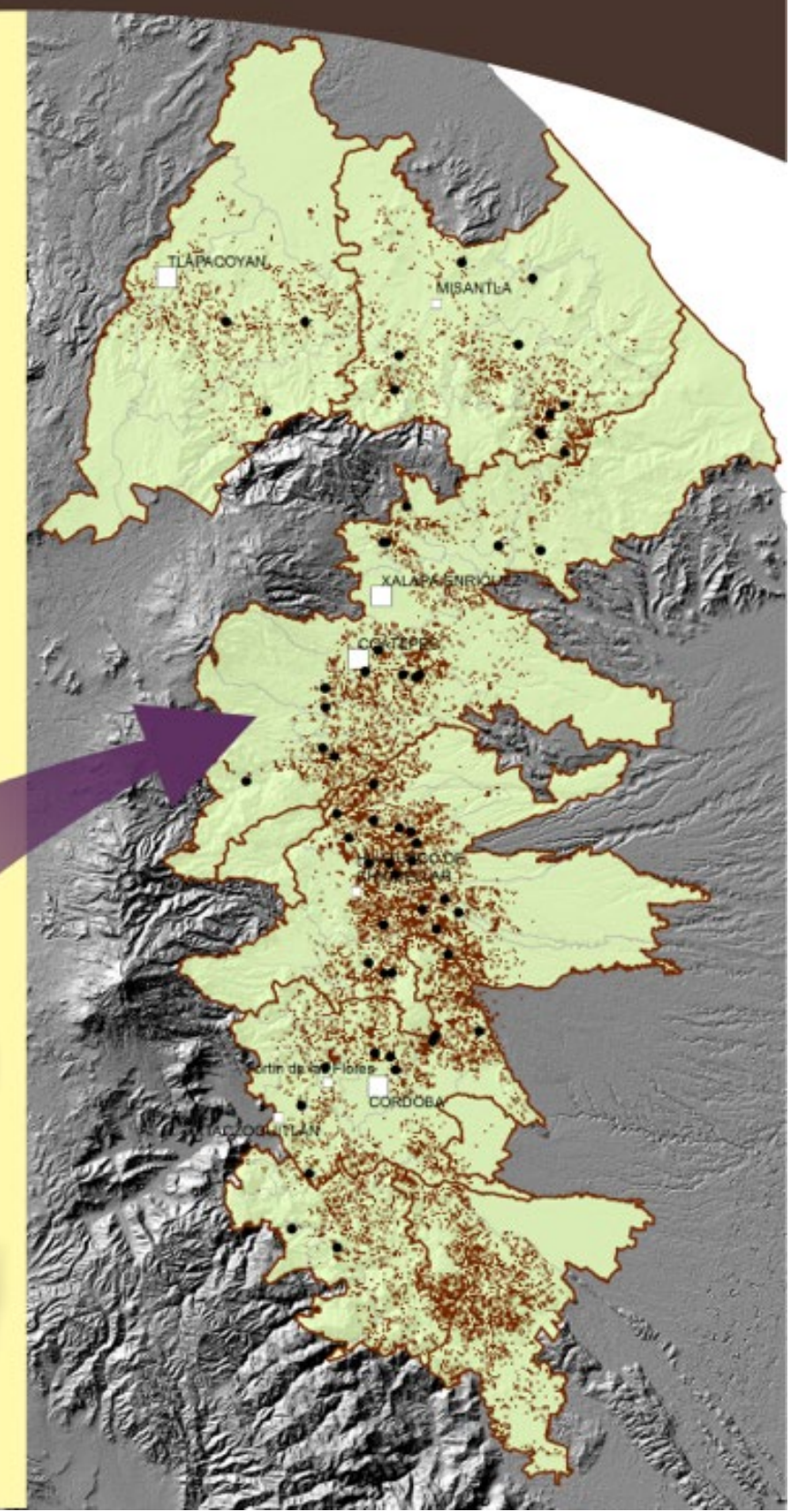

Figura 1. Región cafetalera del centro de Veracruz, México. 
ciones tipo "sello verde"; y mejoramiento del sistema de manejo integral de plagas.

\section{SISTEMAS AGROFORESTALES EN CAFETALES (AGROCAF)}

En esta estrategia se aplican principios agroecológicos y socioeconómicos para el manejo de fincas de café bajo sistemas agroforestales. Se espera a) generar indicadores de tipo experimental e incorporar las experiencias técnicas de productores y organizaciones sociales para el logro de cafetales diversificados en sistemas rústicos y en policultivo; b) reunir la información técnica, científica y saberes campesinos; c) realizar estudios para afinar las recomendaciones de cómo lograr una diversificación agroforestal del café de sombra que preserve el germoplasma forestal de la región y las variedades de café; y d) fomentar la sustentabilidad alimentaria y ampliar el número de productos generados por las fincas de café incluyendo la madera, leña, plantas epífitas de ornato (orquídeas, bromelias, helechos), frutas tropicales, palmilla, entre otras.

\section{CADENAS PRODUCTIVAS DEL CAFÉ (CADECAF)}

Integra las cadenas de producción, procesamiento y comercialización del café, mediante un sistema de trazabilidad para el desarrollo de café sustentable y de alta calidad. Para esto se identificaron los grupos de trabajo (colaboradores de la red) formados por pequeños y medianos productores de café que establecieron alianzas financieras y comerciales. Con estos grupos se generó información sobre el comportamiento del mercado regional, agregar valor al producto, diferenciarlo estratégicamente y desarrollar nuevas marcas y canales de comercialización y distribución. Esto permitió dar ventajas al clúster y ayudar a proyectarlo tanto a nivel nacional como internacional. Se generó y difundió un modelo de innovación y gobernabilidad para el grupo que puede ser referencia en el desarrollo de una política pública para el sector cafetalero.

\section{DESARROLLO EMPRESARIAL Y DE LA CULTURA DEL CAFÉ (EMPRECAF)}

Una tarea importante es realizar diagnósticos empresariales de las fincas cafetalera de la región para: a) impulsar la incubación de emprendimientos rurales de innovación; b) transferir tecnología pertinente, tanto en materia de organización y certificación como en ecotecnias y procesos industriales; c) apoyar la incubación de empresas integradoras que sean necesarias para generar eficiencias colectivas, economías de escala y de aglomeración en la región; d) promover el desarrollo de planes de negocio e inversión, así como planes estratégicos entre los grupos participantes.

Por otro lado, la estrategia busca fortalecer la cultura cafetalera como una forma de articular la vida cotidiana entre sus actores -niños, jóvenes, mujeres y hombres- dedicados al café. Busca rescatar los conocimientos ancestrales y reconocer los procesos globalizados del café. Por ello, la red pretende mostrar la historia local, el valor del trabajo y las formas estéticas de interpretar la organización y los cafetales. A la vez que se proyectan las luchas sociales históricas, se recogen las demandas de los grupos sociales y se discute el futuro, revalorando a los trabajadores del café en la sociedad actual. Estos propósitos se lograrán a través de la educación popular no formal.

\section{RESULTADOS}

La investigación agronómica en el trópico, hasta hace poco, se desempeñó bajo el fundamento ecológico-agronómico propio de las zonas templadas, cuya base es la relación entre una población vegetal y los factores ambientales que la afectan. Sin embargo, un $70 \%$ de la agricultura en el trópico está basada en la biodiversidad, donde el agricultor de pequeñas superficies maneja agroecosistemas complejos con interacciones mutuas entre las especies que lo componen y su entorno (Hart, 1979); la racionalidad del manejo lo regulan las condiciones socioeconómicas y culturales locales. Así, el café en Veracruz, se caracteriza por su alta agrobiodiversidad, cuyo manejo contempla ciertos principios asociados al bosque 
primario que reemplazó. Bajo esta reflexión, una tarea inicial fue la adecuación de bases conceptuales de operación.

La primera fase de ejecución se caracterizó por la integración de saberes, experiencias tácitas y explícitas, capacidades, recursos y necesidades, comprendiendo la serie de actividades programadas en las cinco estrategias. Las actividades fueron: un diagnóstico integral; diseño de experimentos; desarrollo de capacidades humanas e institucionales; transferencia de tecnología; y reorganización productiva. La red en operación interviene coordinadamente con los diferentes actores: productores, empresarios, académicos y representantes de gobierno. Los equipos de trabajo, además de atender los objetivos de investigación, alimentan al sistema de inteligencia con informes de trabajo, documentos de casos exitosos y propuestas. A su vez, los equipos de comunicación elaboran los despachos, informes y boletines que de manera ágil y oportuna se distribuyen a los integrantes de la red. Por su parte, las organizaciones, municipios y actores de la cadena de café analizan la información recibida, la distribuyen en su entorno inmediato y para completar el ciclo, regresan los comentarios al centro de inteligencia.

El diagnóstico integral se abocó a fijar las bases para el centro de inteligencia con las tareas de recolección de información, sistematización y comunicación, que permiten un monitoreo continuo del territorio. El principal desafío es habilitar el soporte informático y técnico para operar la red. Se tienen los registros de los equipos de trabajo, el contacto con organizaciones y el equipo de enlace entre las instituciones académicas y las fincas de estudio. Una de las ventajas de las redes es el soporte tecnológico y la capacidad de registro y documentación de las experiencias. En nuestro caso, las experiencias documentadas son analizadas por un equipo de evaluadores que registra y supervisa el desempeño del proyecto. Un esquema del modelo de funcionamiento de la red, se presenta en la (Figura 2).

El diseño de experimentos agroecológicos requirió de trabajo de campo. Para esto se iniciaron doce estudios que están generando nuevos datos del territorio. La investigación está canalizada a dos áreas: una sobre la diversificación de la producción y los mercados de café, y la otra a los pagos por servicios ambientales en fincas de café de sombra. Los hallazgos realimentan al sistema de inteligencia, y son revisados por los actores en el territorio, en un ciclo de aprendizaje continuo. Para apoyar la comunicación se realizan reuniones del grupo coordinador y operativo del proyecto, se elaboran y distribuyen despachos informativos, vía reuniones presenciales y a través de la página web (http://proyectos.inecol. edu.mx/redcafesustentable/). Estas vías también son utilizadas para enviar comunicados del proyecto a la región, a representantes de otros estados productores de café, a las instituciones del sector y la vinculación con la cadena de comercialización. Desde las fincas recibimos propuestas, sugerencias y necesidades que son discutidas en la comunidad, en reuniones con las organizaciones locales y regionales, y con el grupo operativo del proyecto (Figura 3).

El desarrollo de capacidades humanas e institucionales se fomentó mediante cursos y talleres para la transmisión de conocimiento y para la generación de modelos de aprendizaje multisectorial. La red se enfocó a compartir la cultura del café y los enfoques transdisciplinarios de sustentabilidad entre académicos, productores, técnicos y estudiantes (Figura 4).

La transferencia de tecnología está ligada con los procesos productivos y su incremento competitivo. Se aplican nuevos paquetes tecnológicos y proyectos demostrativos en la región que permitan comparar calidades homogéneas, validar las productividades esperadas, integrar la cadena de valor y mejorar los precios de los productos y servicios. Se busca organizar modelos de gestión que permitan sistematizar socialmente el desempeño de la región y elevar la escala de las acciones en el territorio. El desarrollo y adopción de buenas prácticas es crucial para lograr los objetivos del proyecto. Para esto la red se aboca a conocer, probar y replicar los esquemas de organización, producción y comercialización exitosos.

Se está en proceso de una reorganización productiva que será fundamentada en los diagnósticos empresariales, las capacitaciones y los talleres. 


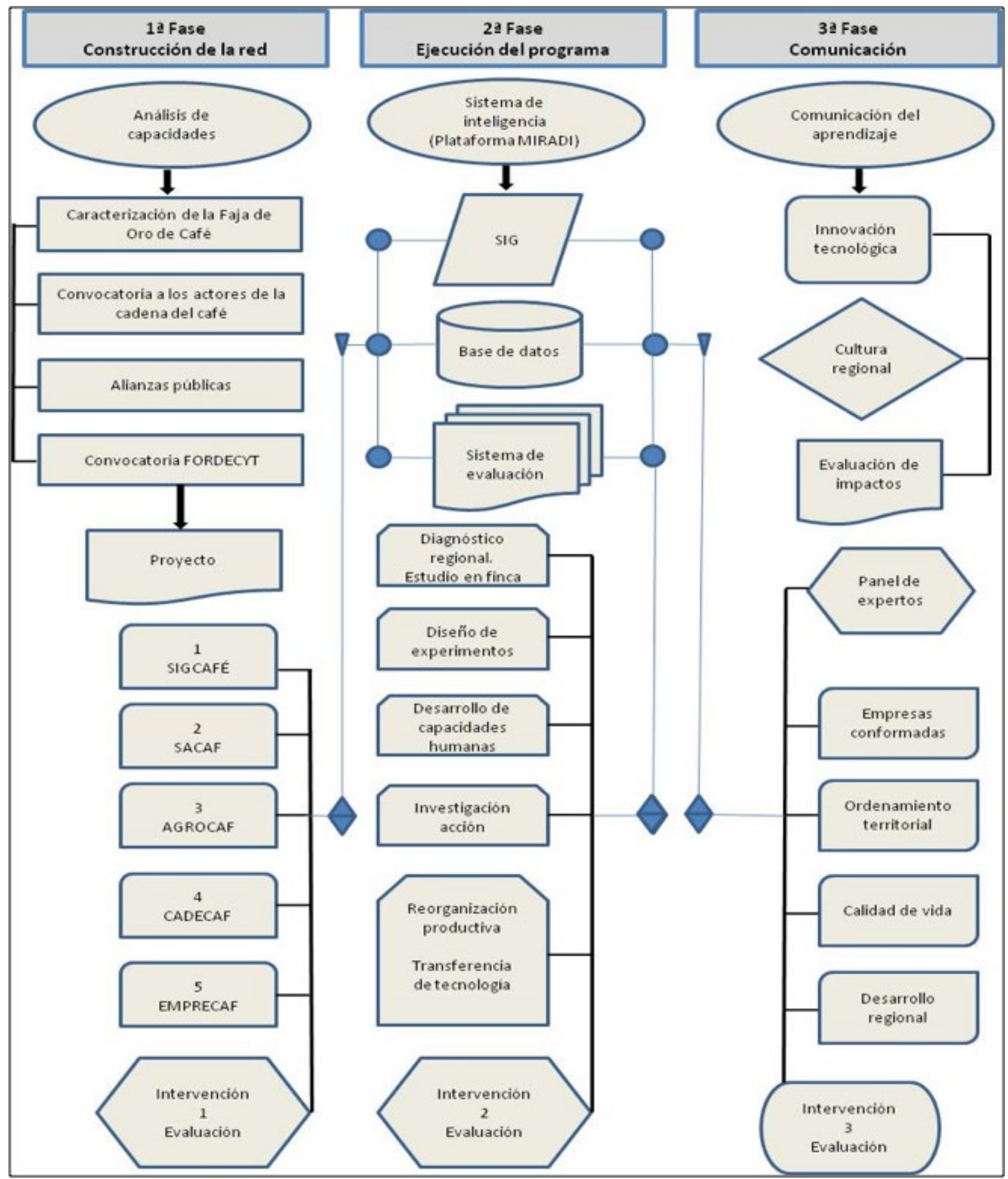

Figura 2. Esquema de la metodología. En la primera fase los acrónimos refieren a las cinco estrategias del proyecto, que incluyen: 1. SIGCAFÉ Sistema de Inteligencia, 2. SACAF Servicios Ambientales, 3. AGROCAF Sistemas Agroforestales, 4. CADECAF Cadena de Valor del Café, 5. EMPRECAF Desarrollo Empresarial y Cultura. 


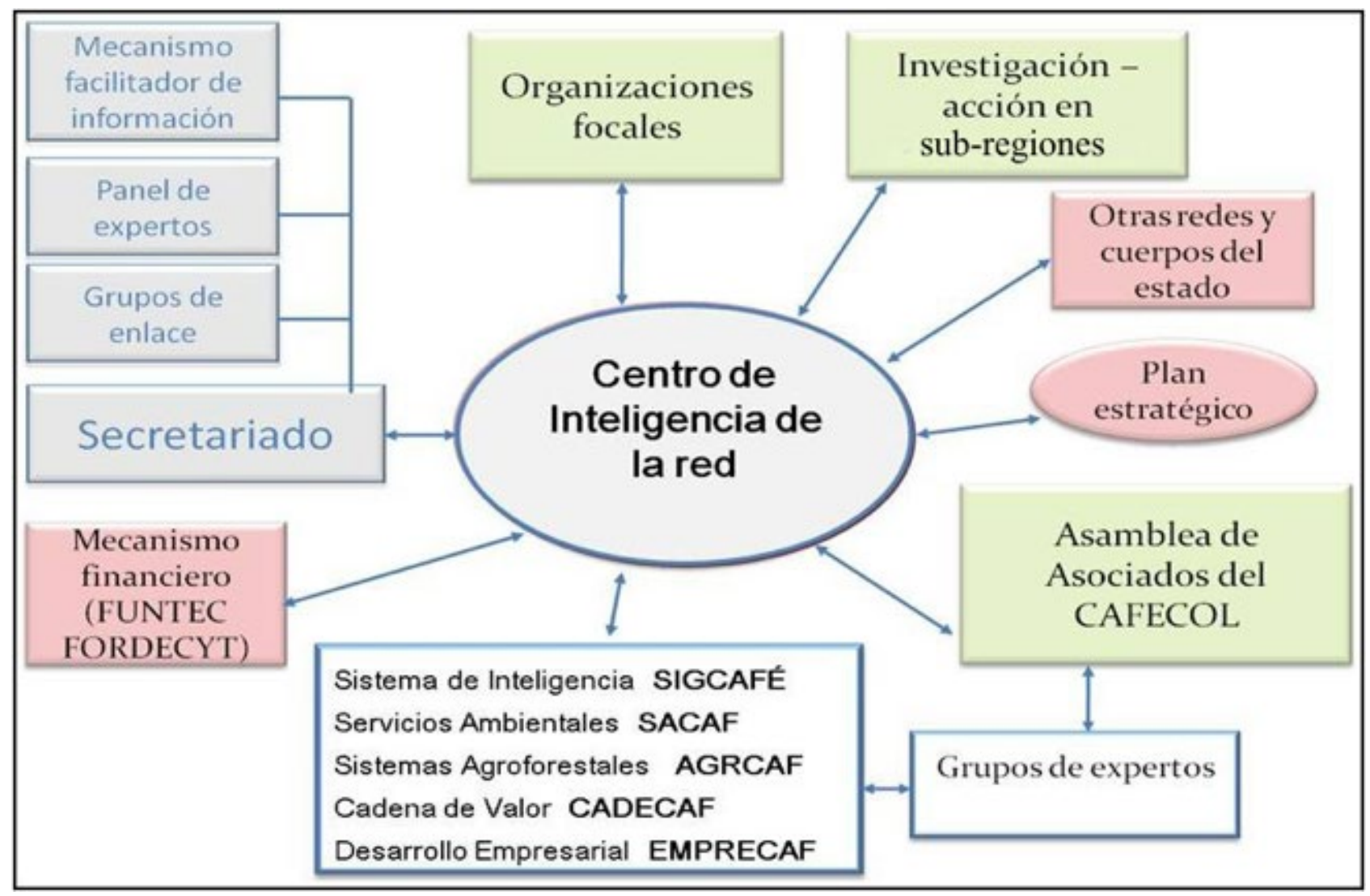

Figura 3. Diagrama de funcionamiento del Centro de Inteligencia
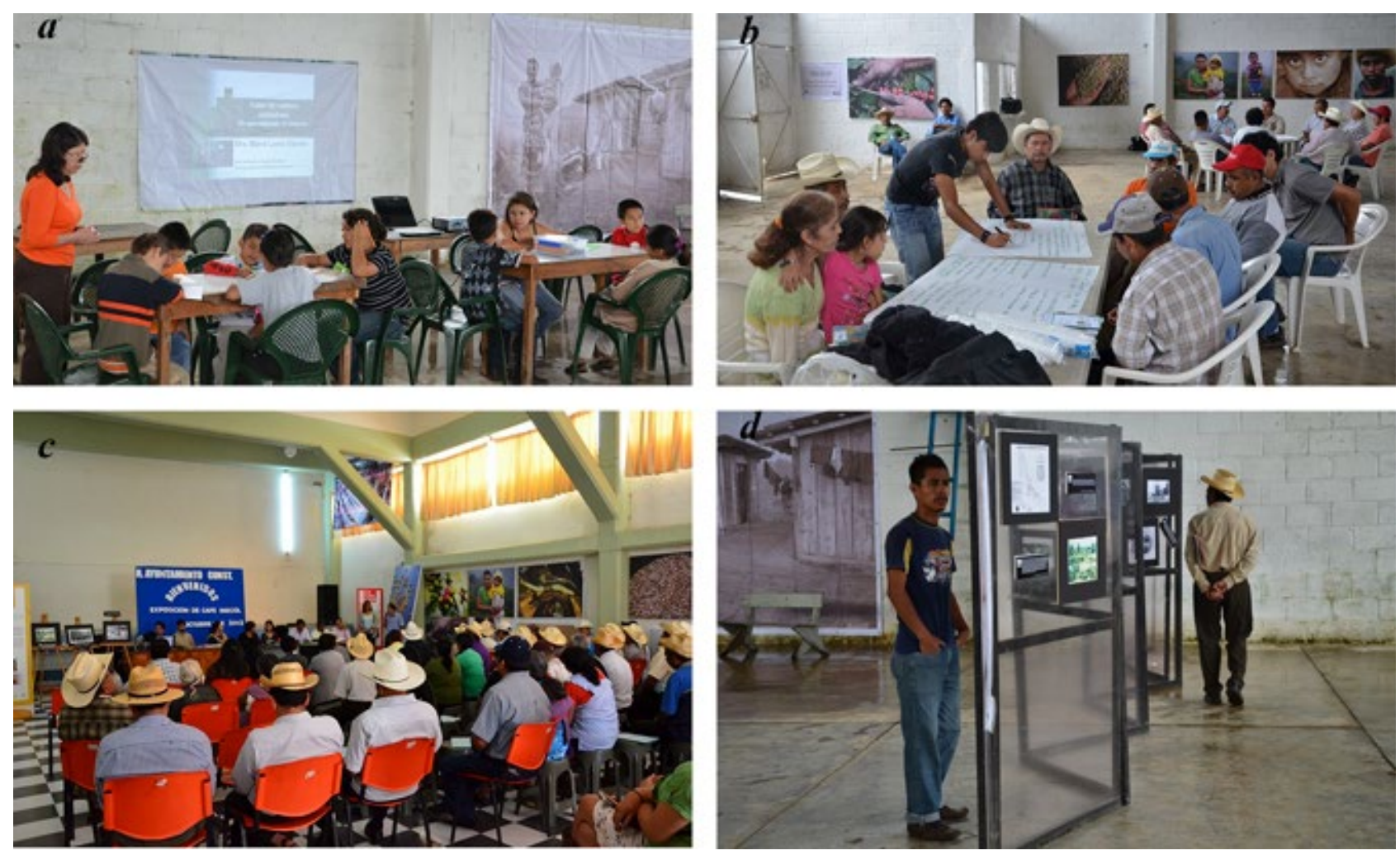

Figura 4. Atención a las capacidades humanas: a) Taller infantil, b) Encuentro en Napoala, c) Taller con productores de Chocamán y d) Exposición en Naopala. 
Evaluaremos los logros del sistema de innovación regional en el clúster cafetalero. Sabemos que la falta de recursos económicos para financiar el cultivo de café es una de las razones del abandono de las fincas $y$ del sector en general. Las organizaciones de productores pondrán en práctica sus valores agregados diferenciados directamente en el mercado y la región, además de los recursos adicionales como su cultura, su potencial y sus productos de especialidad. Con esta diversificación productiva se espera que complementen los ingresos y se mejore el bienestar regional.

Por último, conviene señalar que la red monitorea continuamente el cumplimiento de metas y actividades planteadas, los productos comprometidos y los impactos esperados. Nuestro catálogo de resultados incluyó 44 productos (Cuadro 1). El proyecto tiene una evaluación permanente. Se socializó el proyecto en todos los ámbitos. Para esto organizamos un foro intersectorial al inicio del proyecto y celebramos un segundo foro al término para comunicar los principales logros y experiencias. También, con las acciones del proyecto en referencia, desde un punto de vista sociocultural, integrará grupos informales de productores conformantes de masas críticas que faciliten la transferencia horizontal del conocimiento generado en torno a la cafeticultura del centro de Veracruz. Con cada ciclo de comunicación se ajustan los enfoques al tomar en cuenta los puntos de vista de los productores y las condiciones en que actualmente operan, a fin de contar con una mejor percepción acerca de las oportunidades de investigación, capacitación y difusión cultural en torno a mercados y su relación con la calidad distintiva de nuestro café.

\section{CONCLUSIÓN}

Después de los esfuerzos realizados en el diseño de la red y los apoyos financieros logrados para su ejecución, tenemos la convicción de trabajar junto con los actores de la cadena productiva del café. Por ahora son múltiples los desafíos enfrentados en los primeros tres ańos del proyecto. Es necesario tener apertura para adaptarnos a nuevas formas de trabajo colaborativo, recibimos propuestas y aplicamos iniciativas inéditas.

Nuestra preocupación es consolidar una organización para mejorar el precio del café; a la vez que nos preocupa intervenir en la situación del relevo generacional con la infancia y la juventud de los cafetaleros. Sabemos que sin empleos dignos y estables no será posible la substitución de los talentos de gran experiencia por jóvenes preparados para el trabajo en el campo.

Para el futuro el reto será lograr que la red funcione después del apoyo financiero del FORDECYT, con recursos institucionales renovados y con aportaciones de los propios productores de café. En este sentido, la red tiene el reto de buscar el relevo necesario que con humildad y paciencia, entienda los éxitos y fracasos en el campo del México contemporáneo. 
Cuadro 1. Esquema de estrategias de Café In Red. Catálogo de productos, actividades y principales desafíos.

\begin{tabular}{|c|c|c|c|}
\hline $\begin{array}{l}\text { ESTRA- } \\
\text { TEGIA }\end{array}$ & PRODUCTOS & ACTIVIDADES & DESAFIOS \\
\hline 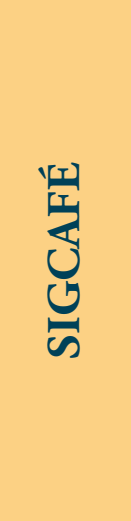 & $\begin{array}{l}\text { Red operativa, bases de datos, } \\
\text { sistema de información geográfica } \\
\text { (SIG),reuniones mensuales con } \\
\text { productores, memoria del trabajo, } \\
\text { sistema de inteligencia, despachos, } \\
\text { boletines, página web, foros de } \\
\text { discusión, talleres de planeación y } \\
\text { evaluación, producción científica, } \\
\text { laboratorio de catación, parcelas } \\
\text { experimentales, informes de políticas } \\
\text { públicas, participación en congresos. }\end{array}$ & $\begin{array}{l}\text { Se organizó la red. Se integraron los } \\
\text { equipos de participantes. Se con- } \\
\text { formó el secretariado para coordinar } \\
\text { las cinco estrategias. Cada estrategia } \\
\text { reporta los avances de sus activida- } \\
\text { des a un coordinador. Se estableció } \\
\text { el observatorio cafetalero, una red de } \\
59 \text { fincas experimentales. }\end{array}$ & $\begin{array}{l}\text { Una institución declinó su partici- } \\
\text { pación porque no cumplió con la } \\
\text { aportación financiera. Hubo que } \\
\text { hacer una restructuración del pro- } \\
\text { yecto para ajustar el plan de trabajo, } \\
\text { los tiempos de los colaboradores y la } \\
\text { administración. }\end{array}$ \\
\hline & $\begin{array}{l}\text { Bases de datos, SIG, talleres de capa- } \\
\text { citación, publicación de manuales, } \\
\text { desarrollo de investigación aplicada, } \\
\text { producción científica, incremento de } \\
\text { colecciones científicas, formación de } \\
\text { recursos humanos. }\end{array}$ & $\begin{array}{l}\text { Se fijaron los criterios para compar- } \\
\text { tir las bases de datos, generar el SIG } \\
\text { compatible con las fuentes actuali- } \\
\text { zadas de información. Se evalúan los } \\
\text { impactos ecológicos y construyen la } \\
\text { propuesta para el esquema de pago } \\
\text { por servicios ambientales (PSA). }\end{array}$ & $\begin{array}{l}\text { Falta mejorar la coordinación entre } \\
\text { dependencias gubernamentales, } \\
\text { fuentes financieras y productores } \\
\text { para mejorar los criterios de PSA. }\end{array}$ \\
\hline$\frac{1}{3}$ & $\begin{array}{l}\text { Bases de datos, SIG, publicación de } \\
\text { libros técnicos, talleres de capaci- } \\
\text { tación, producción de manuales, } \\
\text { producción científica, estudios } \\
\text { forestales, construcción y operación } \\
\text { de viveros, participación en congre- } \\
\text { sos, tesis. }\end{array}$ & $\begin{array}{l}\text { Se elaboran las propuestas para la } \\
\text { diversificación productiva. Se mon- } \\
\text { taron los experimentos y se toman } \\
\text { datos de campo. }\end{array}$ & $\begin{array}{l}\text { Los cambios climáticos impredeci- } \\
\text { bles en los ciclos naturales dificulta } \\
\text { la obtención de datos concluyentes. }\end{array}$ \\
\hline$\sum_{0}^{1}$ & $\begin{array}{l}\text { SIG, talleres de sensibilización } \\
\text { para reforzar la cultura cafetalera, } \\
\text { organización de grupos productivos, } \\
\text { publicación de libros, página web, } \\
\text { encuentros de productores, capaci- } \\
\text { tación, manuales, tesis, gobernanza, } \\
\text { alianzas financieras, mejora del } \\
\text { precio del café, bolsa de producto, } \\
\text { certificación de origen, creación de } \\
\text { marcas locales. }\end{array}$ & $\begin{array}{l}\text { Se trabajó en la difusión de las estra- } \\
\text { tegias de producción, colaboración } \\
\text { y uso de información de la red. Se } \\
\text { evaluó la capacidad de participación } \\
\text { de las organizaciones y se preparan } \\
\text { las condiciones para lograr alianzas } \\
\text { colectivas duraderas. }\end{array}$ & $\begin{array}{l}\text { Los problemas para negociar los } \\
\text { apoyos a la producción del café } \\
\text { limitan la participación colectiva. } \\
\text { Lograr la confianza entre producto- } \\
\text { res y la red requiere de más tiempo; } \\
\text { existe una división entre las asocia- } \\
\text { ciones por su afiliación política. Los } \\
\text { productores están acostumbrados al } \\
\text { asistencialismo. }\end{array}$ \\
\hline 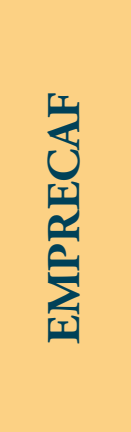 & $\begin{array}{l}\text { Diagnósticos empresariales, publi- } \\
\text { cación de libros, registro audiovisual } \\
\text { de las reuniones con producto- } \\
\text { res, producción de radionovela y } \\
\text { videoclip, talleres infantiles, teatro, } \\
\text { exposiciones fotográficas, encuentros } \\
\text { de tradición oral, talleres de capaci- } \\
\text { tación, manuales, comercio de café, } \\
\text { apoyo a tesis estudiantiles. }\end{array}$ & $\begin{array}{l}\text { El abandono actual de las empresas } \\
\text { (privadas y sociales) demanda inver- } \\
\text { tir en infraestructura básica, capaci- } \\
\text { tación y promoción de tecnología. } \\
\text { La coordinación de los estudios y } \\
\text { los ciclos de producción requiere de } \\
\text { esfuerzos de colaboración. }\end{array}$ & $\begin{array}{l}\text { La participación de fuentes financie- } \\
\text { ras y su continuidad es indispensable } \\
\text { para aplicar soluciones de corto, } \\
\text { mediano y largo plazos para atender } \\
\text { los problemas detectados. Impulsar } \\
\text { la cultura del café requiere de gran } \\
\text { inversión de tiempo y los logros son } \\
\text { de mediano y largo plazo. }\end{array}$ \\
\hline
\end{tabular}




\section{REFERENCIAS}

Bacon, M.C., Mendez, V.E. \& Brown, M.C. (2005) Participatory action-research and support for community development and conservation: examples from shade coffee landscapes of El Salvador and Nicaragua. Research Brief \# 6. Center for Agroecology and Sustainable Food Systems (CASFS), University of California: Santa Cruz, CA, U.S.A. 12 p.

Boege, S. E. (2008) El patrimonio Biocultural de los pueblos indígenas de México: hacia la conservación In situ de la biodiversidad y agrobiodiversidad en los territorios indigenas. Instituto Nacional de Antropología e Historia, Comisión Nacional para el Desarrollo de los Pueblos Indígenas, $342 \mathrm{p}$.

Bruijnzeel, L.A. (2001) Hydrology of tropical montane cloud forests: a reassessment. Land Use and Water Resources Research. 1:1.1-1.18 (www.luwrr.com).

Bubb, P., I. May, L. Miles. \& Sayer, J. (2004) Cloud forest agenda. UNEP-WCMC, Cambridge, UK, 36 p.

Challenger, A. (1998) Utilización y conservación de los ecosistemas terrestres de México. Comisión Nacional para el Conocimiento y uso de la Biodiversidad, México, DF, 847 p.

Contreras, H. A. (2008) Retos en la organización de un proyecto de investigación acción. En: Manson R, V. Hernández-Ortiz, S. Gallina y K. Mehltreter (eds.). 2008. Agroecosistemas cafetaleros de Veracruz: biodiversidad, manejo y conservación. INE, SEMARNAT e INECOL. 35-54 p.

Contreras, H. A., \& Hernández-Martínez, G. (2008) ¡Qué bien huele, mejor sabrá! La organización de productores del proyecto Biocafé. Instituto de Ecología A. C. Xalapa. 91 p.

De Grammont, C. H. (2005) Del desarrollo interno a la globalización: las transformaciones del campo mexicano. $5^{\circ}$ Congreso de la Asociación Mexicana de Estudios Rurales, Oaxaca. Mayo 25 al 28. Balance y perspectivas del campo mexicano, a una década del TLCAN y del Movimiento Zapatista.

De Grammont, C. H. \& Tejeda, H. G. (1996) La Sociedad Rural Mexicana Frente al Nuevo Milenio. Los Nuevos Actores Sociales y Procesos Políticos en el Campo. Ed. Plaza y Valdez. Vol. IV. México, DF.

Drucker, C. R. (2010) "Urge más ciencia y la tecnología: UNAM”. El Universal 27 de abril.
Escamilla, P. E. (2007) Influencia de los factores ambientales, genéticos, agronómicos y sociales en la calidad del café orgánico en México. Colegio de Postgraduados/Campus Veracruz. Tesis de Doctorado. M.F. Altamirano, Veracruz, México. 254 p.

Gordon, E.C., Manson, R.H., Sundberg J. \& Cruz-Angón A. (2007) Biodiversity, Profitability, and Vegetation Structure in Coffee Agroecosystems of central Veracruz, México. Agriculture, Ecosystems and Environment 118: 256-266.

Hart, R.D. (1979) Agroecosistemas: Conceptos Básicos. CATIE, Turrialba, Costa Rica. 211p.

Hernández-Martínez, G., Manson, R.H. \& ContrerasHernández, A. (2009) Quantitative classification of coffee agroecosystems spanning a range of production intensities in central Veracruz, México. Agriculture, Ecosystems \& Environmental 134: 89-98

Manson, H.R., Hernández-Ortiz, V., Gallina T. S. \& Mehltreter, K. (2008) Agroecosistemas cafetaleros de Veracruz: biodiversidad, manejo y conservación. INE, SEMARNAT e INECOL. 330 p.

Moguel, P. \& Toledo, V.M. (1999) Biodiversity conservation in traditional coffee systems of México. Conservation Biology 13(1):11-21.

Morin, E. \& Brigitte, A.K. (1993) Tierra patria. Barcelona: Ed. Kairós. 232 p.

Myers, N. (1997) The world's forests and their ecosystem sevices. In: G. C. Daily (ed.). Natures Services: societal dependence on natural ecosystems. Island Press, Washington, DC. p. 215-235.

Nicolescu, B. (2008) Transdisciplinarity-Theory and practice, Hampton Press, Cresskill, New Jersey. 320 p.

Nolasco, A, M. (1985) Café y Sociedad en México. Centro de Ecodesarrollo, México, DF, 454 p.

Núñez, M.C., Rehaag, I.T., Sánchez, A. \& Vargas, E.M. (2011) Transdisciplinariedad y sostenibilidad: encuentro con Basarab Nicolescu. Universidad Veracruzana. 119 p.

Perfecto, I., Rice, R., Greenburg, R. \& Van Der Voort M. (1996) Shade coffee: A disappearing refuge for biodiversity. Bioscience 46(8): 598-608.

Perfecto, I., Vandermeer, J., MAS, A. \& Soto Pinto L. (2005) Biodiversity, yield, and shade coffee certification. Ecological Economics 54: 435-446. 
Ramamoorthy, T., Bye, R., Lot, A. \& Fa, J. (1993) Biological diversity of México: origins and distribution. Oxford University Press, NY, $812 \mathrm{p}$.

Rzedowski, R.J. (1996) Análisis preliminar de la flora vascular de los bosques mesófilos de montaña de México. Acta Botánica Mexicana 35: 25-44.

Schroth, G., Fonseca, G., Harvey, C., Gascon, C., Vasconcelos, H. \& Izac, A. (2004) Agroforestry and biodiversity conservation in tropical landscapes. Island Press, Washington, DC, $523 \mathrm{p}$.

Stadtmüller, T. \& Agudelo, N. (1990) Amounts and variability of cloud moisture input in a tropical cloud forest. Int. Assoc. Hydrol. Sci. Publ. No. 193, p. 25-32.
Universidad Autónoma de Chapingo (2005) Acciones de fomento productivo y mejoramiento de la calidad del café en México, 2004. Evaluación nacional externa. Universidad Autónoma de Chapingo (CRUO-CENIDERCAFÉ). SAGARPA. Consejo Mexicano del Café, Veracruz. México $104 \mathrm{p}$.

Zermeño, G.S. (2010) Reconstruir a México en el siglo XXI: estrategias para mejorar la calidad de vida y enfrentar la destrucción del medio ambiente. Editorial Océano, 298 p. 